EPJ Web of Conferences 36, 00006 (2012)

DOI: $10.1051 /$ epjconf/20123600006

(C) Owned by the authors, published by EDP Sciences, 2012

\title{
Light vector mesons and the dielectron continuum in PHENIX
}

\author{
Sarah Campbel1 ${ }^{1, \text { a }}$ for the PHENIX Collaboration \\ ${ }^{1}$ Iowa State University
}

\begin{abstract}
The PHENIX experiment at RHIC has measured the dielectron continuum and the $\omega$ and $\phi$ light vector mesons using hadronic and dielectron decay channels in $\sqrt{s_{N N}}=200 \mathrm{GeV} \mathrm{p}+\mathrm{p}, \mathrm{d}+\mathrm{Au}, \mathrm{Cu}+\mathrm{Cu}$ and $\mathrm{Au}+\mathrm{Au}$ collisions. The $\omega$ and $\phi$ mesons experience no strong cold nuclear matter effects but in central heavy ion collisions their yields are suppressed at high $p_{T}$, extending to intermediate $p_{T}$ for the $\phi$. The comparison of the $\omega$ and $\phi$ suppression to the suppression of other hadrons suggests parton energy loss for high $p_{T}$ mesons and favors a recombination production mechanism for intermediate $p_{T} \phi$ mesons. The dielectron continuum shows a low mass excess in central $\mathrm{Au}+\mathrm{Au}$ and $\mathrm{Cu}+\mathrm{Cu}$ collisions while the $\mathrm{p}+\mathrm{p}$ and $\mathrm{d}+\mathrm{Au}$ spectra agree with their respective cocktails. The low mass excess consists of a high $p_{T}$ thermal virtual photon component and a much larger low $p_{T}$ component.
\end{abstract}

\section{Introduction}

Hadrons produced in relativistic heavy ion collisions provide a mechanism to study the quark gluon plasma. This proceeding discusses PHENIX's $\omega$ and $\phi$ light vector mesons measurements from both hadronic and dielectron decays and the dielectron continuum spectra that provides access to the $\rho$ spectral function. The light vector mesons $(\omega, \phi, \rho)$ are predicted to undergo mass and/or width modifications by the medium created in the collision [1] and broadening of the $\rho$ spectral line shape was measured by NA60 at SPS [2]. The dielectron decays of vector mesons provide electro-magnetic signals without color charge that are not modified by the final state and sample a large time window during the collision's evolution. With both the dielectron and hadron decay channels of the $\omega$ and $\phi$, we can extend the transverse momentum, $p_{T}$, range of the measurements and compare decay channels to identify possible variations due to medium effects.

The nuclear modification factor, $R_{A A}$, of the $\omega$ and $\phi$ in heavy ion collisions provides insight into energy loss and particle production mechanisms. $R_{A A}$, Equation 1 , is the ratio of the heavy ion spectrum to a $\mathrm{p}+\mathrm{p}$ reference spectrum scaled by the average number of binary collisions, $N_{\text {Coll }}$.

$$
R_{A A}\left(p_{T}\right)=\frac{d^{2} N_{A A} / d y d p_{T}}{\left(N_{\text {Coll }} / \sigma_{p p}^{\text {inel }}\right) \times d^{2} \sigma_{p p} / d y d p_{T}}
$$

where $d^{2} N_{A A} / d y d p_{T}$ is the differential yield per event in $\mathrm{A}+\mathrm{A}$ collisions, $\sigma_{p p}^{\text {inel }}$ is the total inelastic $\mathrm{p}+\mathrm{p}$ cross-section, and $d^{2} \sigma_{p p} / d y d p_{T}$ is the differential $\mathrm{p}+\mathrm{p}$ cross-section. In heavy ion collisions, a factor of five suppression of the $\pi^{0}$ and $\eta$ meson production with respect to the $\mathrm{p}+\mathrm{p}$ scaled by the average number of binary collisions is seen [3] [4]. Protons [5] and other baryons [6] [7] do not experience this suppression particularly at intermediate $p_{T}$ where they are enhanced relative to binary scaled $\mathrm{p}+\mathrm{p}$ collisions. Since the $\omega$ meson has the same quark content as the $\pi^{0}$ but with a larger mass and intrinsic spin, it's behavior can provide more information on energy loss in the quark gluon plasma. The $\phi$ meson is a $s \bar{s}$ state with a mass similar to the proton, it can also answer questions of energy loss and baryon-meson differences.

\footnotetext{
a e-mail: campbels@iastate.edu
}

This is an Open Access article distributed under the terms of the Creative Commons Attribution License 2.0, which permits unrestricted use, distribution, and reproduction in any medium, provided the original work is properly cited. 


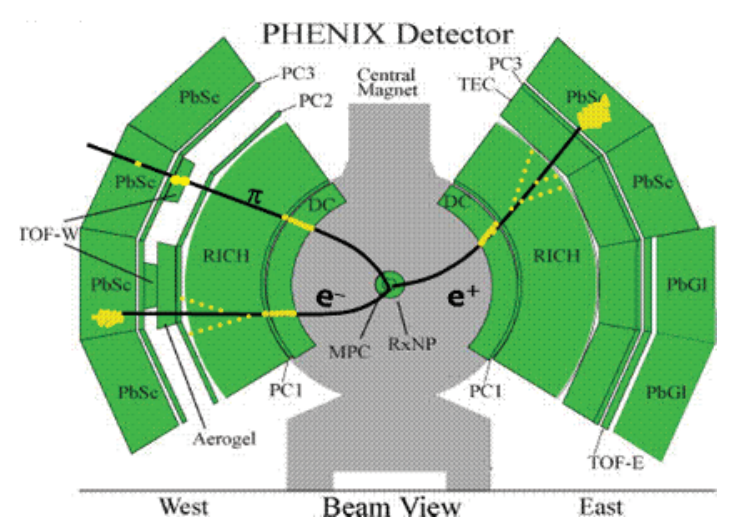

Fig. 1. The beam view of the central arms of the PHENIX detector with example tracks for a positron, an electron, and a charged pion.

First the PHENIX central arm detectors are presented. Then PHENIX $\omega$ and $\phi$ measurements are discussed. This is followed by PHENIX's dielectron continuum measurements and a brief conclusion.

\section{PHENIX detector}

The central arms of the PHENIX detector, shown in Figure 1, consist of two spectrometers each with spanning $\pi / 2$ in azimuth and \pm 0.35 in pseudorapidity. Charged particles bent by the magnetic field are tracked in the drift chamber (DC) and first pad chamber (PC1) with a momentum resolution of $\delta p / p=0.7 \% \oplus 1.1 \% p[\mathrm{GeV} / \mathrm{c}][8]$. Two electro-magnetic calorimeters (PbSc and $\mathrm{PbGl}$ ) measure photons and reconstruct photon pairs from $\pi^{0}$ and $\eta$ decays. Electrons are identified by a Cherenkov ring in the ring imaging Cherenkov detector $(\mathrm{RICH})$ and an electro-magnetic shower in the $\mathrm{PbGl}$ and $\mathrm{PbSc}$. Particle identification of charged hadrons is possible using the momentum from the DC and $\mathrm{PC} 1$ and the time of flight information from the time-of-flight (TOF) and aerogel detectors. The TOFEast is used for kaon identification in the $\phi \rightarrow K^{+} K^{-}$channel presented in Section 3. It has a time resolution of $120 \mathrm{ps}$ and a $\pi / \mathrm{K}$ separation in the $p_{T}$ range $0.3-2.2 \mathrm{GeV} / \mathrm{c}$ [9]. Beam-beam counters and zero degree calorimeters are located around the beam pipe to the north and south of the central arms [10]. They determine the position of the collision along the beam axis and the collision's centrality, a measure of the collision's nuclear overlap.

\section{The $\phi$ and $\omega$ mesons}

The $\phi$ and $\omega$ mesons are measured in $s_{N N}=\sqrt{200} \mathrm{GeV} p+\mathrm{p}, \mathrm{d}+\mathrm{Au}, \mathrm{Cu}+\mathrm{Cu}$ and $\mathrm{Au}+\mathrm{Au}$ collisions with a variety of decay channels, presented in Table 1. Raw yields are corrected for the acceptance and reconstruction efficiency, embedding efficiency to correct for occupancy effects, trigger bias or efficiency effects, and the decay's branching ratio. By measuring the $\phi$ and $\omega p_{T}$ spectra in different channels the $p_{T}$ range is extended. A smooth transition from the dielectron decays at low $p_{T}$ to the hadronic decays at high $p_{T}$ is seen with overlap in the intermediate $p_{T}$ range where they agree. This agreement is seen in the $\phi$ from 1 to $3 \mathrm{GeV} / \mathrm{c}$ in $\mathrm{p}+\mathrm{p}$ collisions, from 1 to $5 \mathrm{GeV} / \mathrm{c}$ in $\mathrm{d}+\mathrm{Au}$ [11] and $\mathrm{Au}+\mathrm{Au}$ collisions and in the $\omega$ from 2 to $4 \mathrm{GeV} / \mathrm{c}$ in $\mathrm{p}+\mathrm{p}$ collisions and from 2 to $6 \mathrm{GeV} / \mathrm{c}$ in $\mathrm{d}+\mathrm{Au}$ collisions [12].

\subsection{Nuclear modification}

$\mathrm{d}+\mathrm{Au}$ collisions provide access to initial state cold nuclear matter effects, while heavy ion collisions such as $\mathrm{Cu}+\mathrm{Cu}$ and $\mathrm{Au}+\mathrm{Au}$ generate additional medium effects from the quark gluon plasma. The nuclear modifications of $\mathrm{d}+\mathrm{Au}, \mathrm{Cu}+\mathrm{Cu}$ and $\mathrm{Au}+\mathrm{Au}$ spectra are calculated by taking the ratio to the 
Table 1. $\phi$ and $\omega$ meson decays measured in various collision systems.

\begin{tabular}{llll}
\hline $\mathrm{p}+\mathrm{p}$ & $\mathrm{d}+\mathrm{Au}$ & $\mathrm{Cu}+\mathrm{Cu}$ & $\mathrm{Au}+\mathrm{Au}$ \\
\hline$\phi \rightarrow e^{+} e^{-}$ & $\phi \rightarrow e^{+} e^{-}$ & $\phi \rightarrow e^{+} e^{-}$ & $\phi \rightarrow e^{+} e^{-}$ \\
$\phi \rightarrow K^{+} K^{-}$ & $\phi \rightarrow K^{+} K^{-}$ & $\phi \rightarrow K^{+} K^{-}$ & $\phi \rightarrow K^{+} K^{-}$ \\
$\omega \rightarrow e^{+} e^{-}$ & $\omega \rightarrow e^{+} e^{-}$ & $\omega \rightarrow e^{+} e^{-}$ & $\omega \rightarrow e^{+} e^{-}$ \\
$\omega \rightarrow \pi^{0} \gamma$ & $\omega \rightarrow \pi^{0} \gamma$ & $\omega \rightarrow \pi^{0} \gamma$ & $\omega \rightarrow \pi^{0} \gamma$ \\
$\omega \rightarrow \pi^{+} \pi^{-} \pi^{0}$ & $\omega \rightarrow \pi^{+} \pi^{-} \pi^{0}$ & & \\
\hline
\end{tabular}

p+p spectrum scaled by $N_{\text {Coll }}$, where $N_{\text {Coll }}$ is calculated using a Glauber Monte Carlo simulation. When there are no nuclear effects this nuclear modification factor, $R_{A A}$ (Equation 1) or $R_{d A}$, is one. A nuclear enhancement generates values greater than one and a suppression leads to values of less than one. The $R_{d A}$ quantizes the cold nuclear matter effects in d+Au collisions relative to $N_{C o l l}$-scaled p+p. Figure 2 shows the $R_{d A}$ as a function of $p_{T}$ for $\omega$ and $\phi$ mesons in central 0-20\%, peripheral 60-88\% and minimum bias (MB) d+Au collisions. For comparison these are shown with $R_{d A}$ for $\pi^{0}, \eta, \eta{ }^{\prime}, K_{S}$ and protons. No strong cold nuclear matter effects are seen for the $\omega$ or $\phi$ mesons. No meson species dependence of $R_{d A}$ is seen within the uncertainties. The peripheral d+Au meson spectra are consistent with the $N_{\text {Coll }}$ scaled $\mathrm{p}+\mathrm{p}$ spectra. For central d+Au collisions at intermediate $p_{T}$, there is little to no Cronin enhancement of $\omega, \phi$ and $\pi^{0}$ mesons. A small suppression of $\omega$ and $\pi^{0}$ at $p_{T}$ above $8 \mathrm{GeV} / \mathrm{c}$ is seen. The high $p_{T} R_{d A}$ behavior of the $\omega$ and $\pi^{0}$ mesons suggests that there may be a small energy loss at the parton level given the similarity of the $R_{A A}$ despite different meson masses [12].

Heavy ion collisions consider $R_{A A}$ to identify modifications from the hot dense plasma created in the collision. Figure 3 presents the $R_{A A}$ of $\phi$ and $\omega$ mesons in central $\mathrm{Au}+\mathrm{Au}$ collisions as a function of $p_{T}$. The $\pi^{0}, \eta, K^{+}, \mathrm{J} / \psi$, direct photon, proton and non-photonic electrons are shown for comparison. $\pi^{0}$ and $\eta$ mesons have a factor of five suppression at high $p_{T}$. At $p_{T}$ above $5 \mathrm{GeV} / \mathrm{c}$, the $\phi$ and $\omega$ mesons show a flat suppression coincident with $\pi^{0}$ suppression. As the $p_{T}$ is lowered the $R_{A A}$ of the $\phi$ increases, the intermediate $p_{T}$ behavior of the $\phi$ is similar to what is seen for $K^{+}$, another strange quark meson [5].

The suppression patterns seen in the central $\mathrm{Au}+\mathrm{Au}$ collisions are also apparent in mid-central $\mathrm{Au}+\mathrm{Au}$ and $\mathrm{Cu}+\mathrm{Cu}$ collisions. The $R_{A A}$ as a function of $p_{T}$ in $\mathrm{Cu}+\mathrm{Cu}$ and $\mathrm{Au}+\mathrm{Au}$ collisions are compared for systems with the same number of participating nucleons, $N_{\text {Part }}$. The suppression of the $\omega$ at high $p_{T}$ agrees with the level of $\pi^{0}$ suppression in both $\mathrm{Cu}+\mathrm{Cu}$ and $\mathrm{Au}+\mathrm{Au}$ collisions at intermediate $N_{\text {Part }}$. Similarly, in intermediate $N_{\text {Part }} \mathrm{Cu}+\mathrm{Cu}$ and $\mathrm{Au}+\mathrm{Au}$ collisions, the $\phi$ suppression at intermediate $p_{T}, 2-5 \mathrm{GeV} / \mathrm{c}$, remains above the $\pi^{0}$ and below the proton. At $p_{T}$ above $5 \mathrm{GeV} / \mathrm{c}$ for the same collisions, the $\phi$ suppression agrees with the $\pi^{0}$ suppression. For both intermediate $p_{T} \phi$ and high $p_{T} \omega$ the $N_{\text {Part }}$ dependence of the integrated $R_{A A}$ is consistent in $\mathrm{Cu}+\mathrm{Cu}$ and $\mathrm{Au}+\mathrm{Au}$ collisions despite different collision geometries.

In peripheral collisions, $\omega$ and $\phi$ mesons are consistent with binary scaling. The $\phi$ is also consistent with a small enhancement seen in the peripheral proton $R_{A A}$ [11]. The integrated $R_{A A}$ for high $p_{T} \omega$ mesons as a function of $N_{\text {Part }}$ shows that the suppression starts at a $N_{\text {Part }}$ of 34 and increases with $N_{\text {Part }}$, a trend similar to the $\pi^{0}$ and $\eta$ suppression. This high $p_{T} R_{A A}$ vs $N_{\text {Part }}$ dependence is fit with a fractional energy loss function, $R_{A A}=\left(1-S_{0} N_{\text {Part }}^{a}\right)^{n-2}$, with the n parameter fixed to eight. The resulting $a$ parameter is consistent with PQM [13] and GLV [14] model predictions of a roughly $N_{\text {Part }}^{2 / 3}$ dependence. The similar suppression patterns seen in $\pi^{0}, \eta$ and $\omega$ mesons at high $p_{T}$ suggests that energy loss in the medium occurs at the parton level [15].

Understanding the intermediate $\phi$ suppression behavior is more difficult. Radial flow effects are unlikely causes of the $\phi$ suppression because of the different behaviors of the $\phi$ and proton which have similar masses, while the $\phi$ and $K^{+}$are similarly suppressed even though kaons have less mass. Recombination may account for the $\phi$ and $K^{+}$behavior because of their strangeness content. Given strangeness enhancement, there may be more thermal partons in strange meson production in a recombination scenario. This could result in an extension of soft processes out to higher $p_{T}$ for strange mesons [11]. 


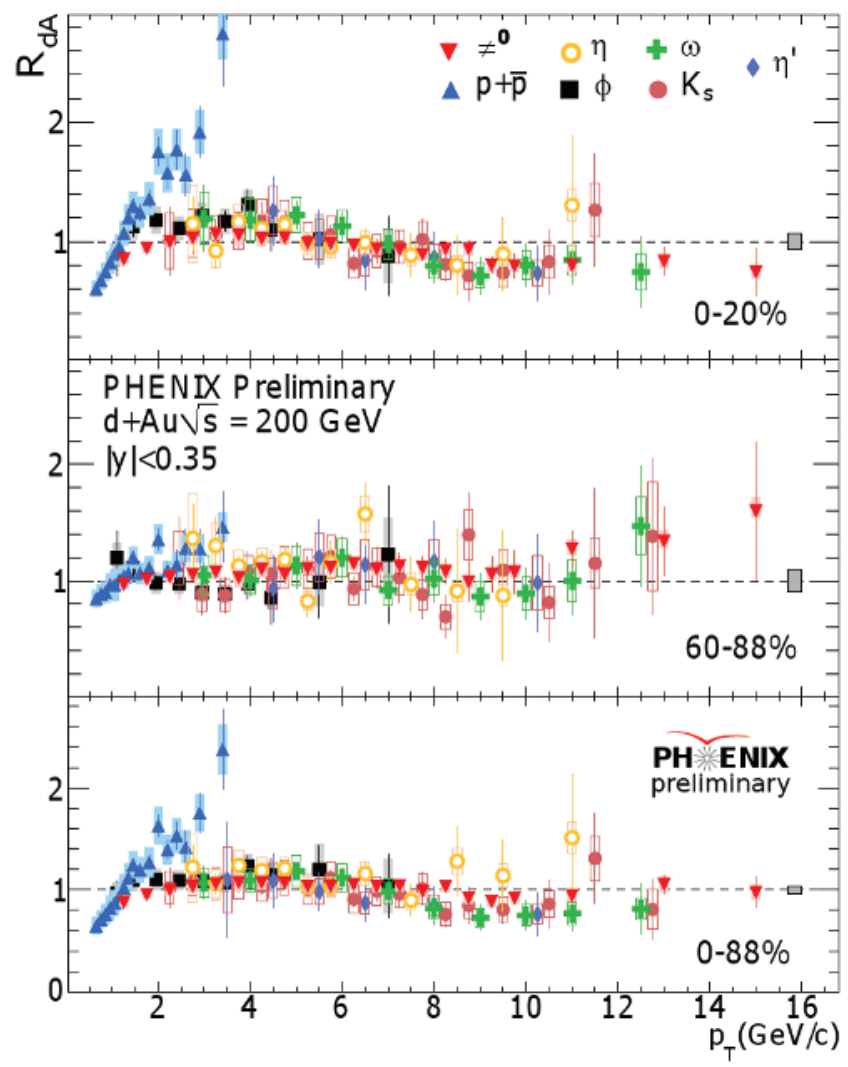

Fig. 2. The $R_{d A}$ vs $p_{T}$ for particles in $0-20 \%, 60-88 \%$ and MB (i.e. $0-88 \%$ ) d+Au collisions. $\pi^{0}, \eta, \eta^{\prime}, \omega, \phi, K_{S}$ and $p+\bar{p}$ are shown. The grey band around one provides the systematic error on the $R_{d A}$ from the uncertainty in $N_{\text {Coll }}$ for each plot.

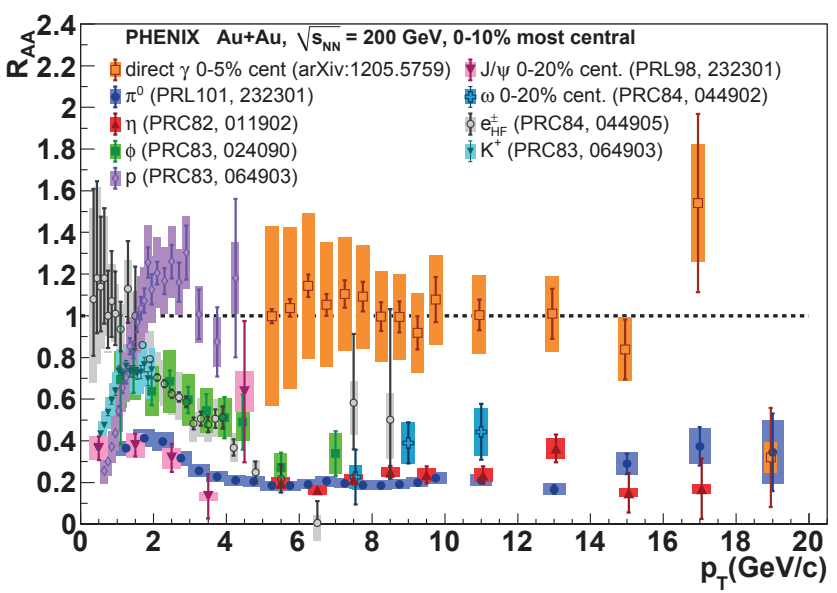

Fig. 3. The $R_{A A}$ vs $p_{T}$ for various particles in $\mathrm{Au}+\mathrm{Au}$ central collisions. The $\pi^{0}$, $\eta, \omega, \phi, K^{+}$, proton, $J / \psi$, direct $\gamma$ and non-photonic electrons or $e_{H F}^{ \pm}$are shown. These particles are from $0-10 \% \mathrm{Au}+\mathrm{Au}$ collisions except for the direct photons which are from $0-5 \% \mathrm{Au}+\mathrm{Au}$ and the $\omega$ and $\mathrm{J} / \psi$ which are from $0-20 \% \mathrm{Au}+\mathrm{Au}$. 


\section{Dielectron continuum}

NA60 measured the dielectron continuum in $158 \mathrm{AGeV}$ In+In collisions at SPS and found the $\rho$ to be broadened without a mass shift [2]. The $\rho$ is a prime signal to identify mass modifications because of it's short lifetime, $2.1 \mathrm{fm} / \mathrm{c}$. As a consequence, the $\rho$ meson is more likely to decay in the medium. In a dielectron decay, an the electron-positron pair produced in medium will escape with no final state effects because leptons contain no color charge. However, the short lifetime of the $\rho$ also means that it has a broad mass distribution. This combined with the decays of neighboring mesons make it difficult to isolate the $\rho$ meson in a mass spectrum. By measuring the dielectron continuum, deviations in the mass spectrum due to $\rho$ modification can be identified. Since the dielectron continuum contains information collected over the full time evolution of the medium, additional physics signals are present in the dielectron continuum. These include direct virtual photons, hadron Dalitz $\left(\pi^{0}, \eta, \eta^{\prime}\right)$ and leptonic $(\omega, \phi, \mathrm{J} / \psi)$ decays, and correlations from electron-positron pairs resulting from semi-leptonic decays of open heavy flavor mesons correlated by the pair production of heavy quarks.

\subsection{Cocktail}

A reference cocktail of the expected hadronic sources is generated to compare the data to the expected physics signals. This is done using EXODUS, a hadronic decay generator. EXODUS handles the decay kinematics for two- and three-body hadron decays and the dielectron pair spectrum is determined. It is parameterized with measured PHENIX data including meson-to-pion ratios and a fit to the pion $p_{T}$ spectrum with a modified Hagedorn exponential, Equation 2 where A, a, b, n, and $p_{0}$ are fit parameters [16].

$$
E \frac{d^{3} \sigma}{d p^{3}}=A\left(e^{-\left(a p_{T}+b p_{T}^{2}\right)}+p_{T} / p_{0}\right)^{-n}
$$

The $p_{T}$ spectra of the other mesons are extrapolated assuming $m_{T}$ scaling. The $m_{T}$ scaling assumption is confirmed for the $\phi$ but deviations are seen at the higher mass $\mathrm{J} / \psi$; the measured $\mathrm{J} / \psi p_{T}$ distribution is used instead. Additionally, meson-to-pion ratios are adjusted to reflect the suppression measured in heavy ion collisions and the $\mathrm{J} / \psi$ and $\psi$ ' line shapes are altered to include radiative tails. The PHENIX cocktail includes electron-positron pairs from vacuum $\rho$ decays; additional dielectron yields due to $\rho$ production via $\pi-\pi$ annihilation are not included.

Dielectron correlations in the mass spectrum from heavy quarks and Drell-Yan are calculated with PYTHIA. In the $\mathrm{p}+\mathrm{p}$ case, the dielectron spectrum is precise enough to subtract the hadronic components and measure the charm cross section. A charm cross section of $544 \pm 39($ stat $) \pm 142($ sys $) \pm$ 200 (model) $\mu b$ was determined by fitting the PYTHIA line shapes for the charm and bottom and Drell-Yan components [17]. This agrees with the non-photonic single electron cross section of $567 \pm$ $57($ stat $) \pm 193($ sys $) \mu b$ [18]. The heavy quark and Drell-Yan line shapes in $\mathrm{p}+\mathrm{p}$ are scaled by the average number of collisions, $N_{\text {Coll }}$, for $\mathrm{d}+\mathrm{Au}, \mathrm{Cu}+\mathrm{Cu}$ and $\mathrm{Au}+\mathrm{Au}$ collisions. As a comparison a randomly correlated $c-\bar{c}$ spectrum is also generated using the PYTHIA single electron $p_{T}$ spectrum and a randomly oriented opening angle between the electron-positron pairs [16]. The hadronic components from EXODUS and the heavy quark and Drell-Yan correlations are filtered in to the ideal PHENIX acceptance.

\subsection{Analysis}

To measure the dielectron continuum, backgrounds pairs must be removed. Backgrounds from conversions and detector effects such as RICH ring overlap are removed with pair cuts such as the $\phi_{V}$ cut and a ring separation cut respectively [16]. The remaining three backgrounds cannot be removed with cuts, they are statistically subtracted. The largest background source is the combinatorial background; the shaped of the combinatorial background is generated for like- and unlike-sign pairs by event mixing using a rolling buffer method. Background pairs from double Dalitz decays and hadronic decays with multiple electron-positron pairs create combinatorial pairs that are correlated from the original 
parent hadron and are not included in the hadronic cocktail generated by EXODUS. These pairs, from pion double Dalitz decay and $\eta \rightarrow \pi \pi \pi$ decays with multiple pion Dalitz decays, are considered background and can be statistically subtracted from the analysis. The like- and unlike-sign line shapes of these decays are simulated using an EXODUS-like code that is able to make pairs from multiple electron-positron pair decay chains. Jets are the remaining source of background since multiple pions within a jet may Dalitz decay creating lepton pairs that are correlated through the jet kinematics. The jet background is simulated using PYTHIA. Both the jet and double Dalitz backgrounds create unlikeand like-sign pairs at the same rates as a result of the pair production of leptons.

The combinatorial, double Dalitz and jet backgrounds can be removed with either of two subtraction methods. This first method is a component by component subtraction that uses the like-sign pairs to find the relative contributions of the three remaining backgrounds. The simulated backgrounds are then subtracted from unlike-sign spectrum. The second background subtraction method is like-sign subtraction where an acceptance correction has been applied to the like-sign pairs. This is done by adjusting the like-sign pair spectrum differentially in mass and pair $p_{T}$ to mimic the unlike-sign pair acceptance and then subtracting the like-sign pairs from the unlike-sign pairs. In $p+p, C u+C u$ and $\mathrm{Au}+\mathrm{Au}$ these two subtraction methods are performed separately and are consistent. The $\mathrm{d}+\mathrm{Au}$ results are obtained with the acceptance corrected like-sign subtraction method.

After the backgrounds are subtracted the spectrum is corrected for the reconstruction efficiency and gaps in the ideal PHENIX acceptance. In the $\mathrm{p}+\mathrm{p}$ and $\mathrm{d}+\mathrm{Au}$ spectra a trigger efficiency correction is also applied.

\subsection{Results}

The dielectron mass spectra in $s_{N N}=\sqrt{200} \mathrm{GeV} \mathrm{MB} \mathrm{d}+\mathrm{Au}$ and $\mathrm{Au}+\mathrm{Au}$ collisions are shown with their cocktails in Figure 4. The $\mathrm{p}+\mathrm{p}$ and $\mathrm{d}+\mathrm{Au}$ spectra agree with the cocktail within the systematic errors. No new cold nuclear matter effects are seen in the MB d+Au dielectron spectrum [19]. The $\mathrm{MB} \mathrm{Au}+\mathrm{Au}$ spectrum contains an enhancement above the cocktail in the low mass region, 0.15-0.75 $\mathrm{GeV} / c^{2}$, by a factor of $4.7 \pm 0.4($ stat $) \pm 1.5(s y s)$ [16]. Below each of these spectra the ratio of the data to the cocktail is shown. This ratio verifies that the known signals are in good agreement with the cocktail, particularly for the $\pi^{0}$ and the $\mathrm{J} / \psi$ where the signal-to-background ratio is large. Often when the $\pi^{0}$ or $\mathrm{J} / \psi$ are not in agreement with the cocktail this is the result of incorrect efficiency and dead area corrections that effect the entire spectrum.

The $0-10 \% \mathrm{Cu}+\mathrm{Cu}$ and $0-10 \%$ and $10-20 \% \mathrm{Au}+\mathrm{Au}$ show an excess above the cocktail in the low mass region providing information on the onset of the excess [20]. Figure 5 allows for a shape comparison of the excess in $0-10 \% \mathrm{Cu}+\mathrm{Cu}$ and $0-10 \%$ and $10-20 \% \mathrm{Au}+\mathrm{Au}$ by overlaying these spectra scaled by $1 / N_{\text {Part }}$. This excess increases at a rate faster than $N_{\text {Part }}^{2}[16]$.

To further study the dielectron continuum, the mass spectrum is considered in different $p_{T}$ ranges, as seen in Figure 6. The $\mathrm{p}+\mathrm{p}$ spectrum at low $p_{T}$ matches the cocktail well. The $\mathrm{MB} \mathrm{Au}+\mathrm{Au}$ and $0-10 \% \mathrm{Cu}+\mathrm{Cu}$ (not shown) spectra show the majority of the low mass excess is located at low pair $p_{T}$. At high $p_{T}$ and intermediate mass, between the $\phi$ and $\mathrm{J} / \psi$, the $\mathrm{p}+\mathrm{p}, \mathrm{Cu}+\mathrm{Cu}$ and $\mathrm{Au}+\mathrm{Au}$ data points are above the cocktail with the PYTHIA $c-\bar{c}$ component (solid line) and the cocktail with the randomly associated $c-\bar{c}$ component (dash-dot line). This discrepancy may be because the single electron $p_{T}$ spectra does not agree with the PYTHIA $p_{T}$ spectra, because the $b-\bar{b}$ component is not included, or because of additional thermal photon contributions. At low mass and high $p_{T}$ a slight excess in $\mathrm{p}+\mathrm{p}$ and larger excesses in the $\mathrm{Au}+\mathrm{Au}$ and $\mathrm{Cu}+\mathrm{Cu}$ spectra are seen. Since the mass of the pair is much smaller than the pair $p_{T}$, this excess may be attributed to virtual thermal photons that convert into dielectron pairs.

Virtual photons are produced in the same processes that generate real photons. When the mass is much less than the $p_{T}$, a virtual thermal photon component follows, $d^{2} N_{e e} / d m_{e e} d p_{T} \propto 1 / m_{e e}$ $d N_{\gamma} / d p_{T}$. The thermal photon component is modeled in EXODUS, filtered into the PHENIX acceptance including dead areas and smeared by the PHENIX resolution. The thermal photon contribution is obtained by fitting the data in the mass region, $0.15-0.3 \mathrm{GeV} / c^{2}$, for different $p_{T}$ slices above $1 \mathrm{GeV} / \mathrm{c}$ with a combination of the thermal photonic component and the cocktail. This is done for the $\mathrm{p}+\mathrm{p}$, 

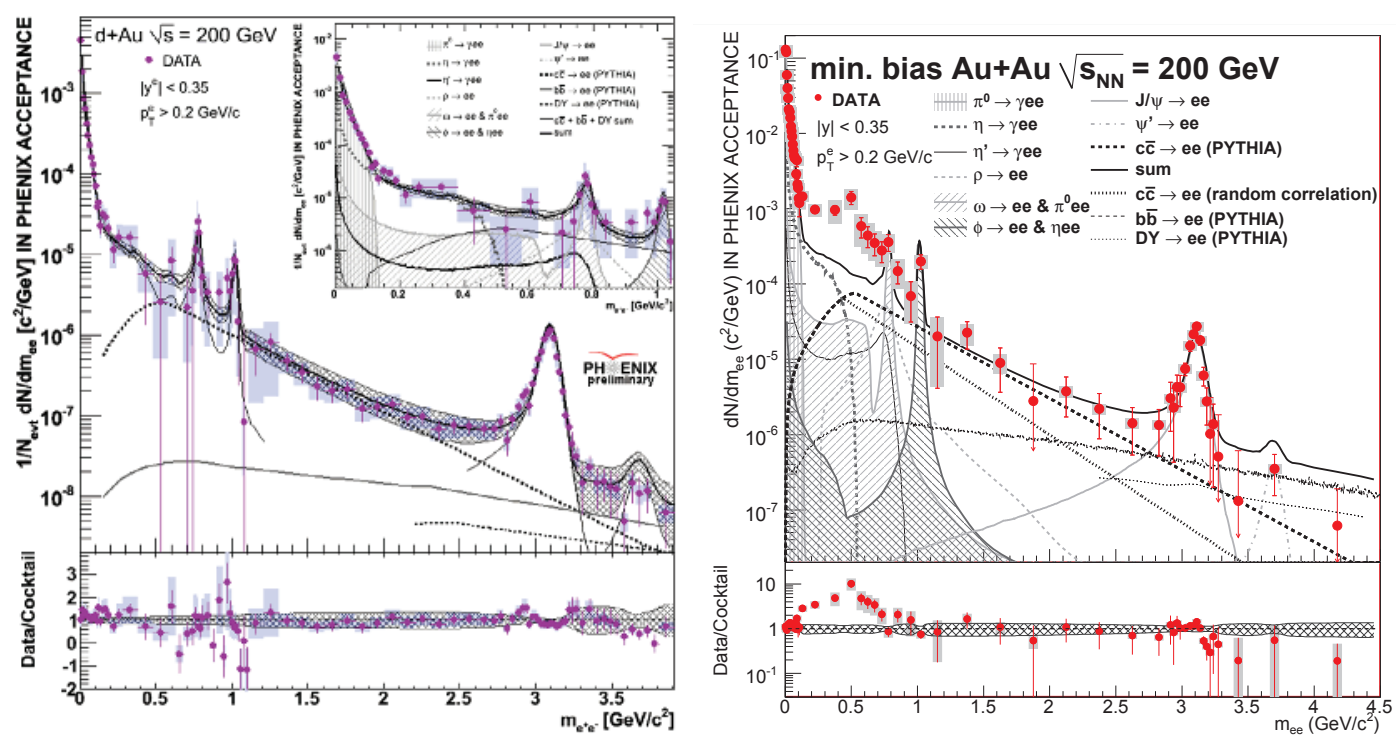

Fig. 4. The MB d+Au and $\mathrm{Au}+\mathrm{Au}$ dielectron spectra compared to their respective cocktails. Under each plot the ratio of the data to the cocktail is shown.[16][19]

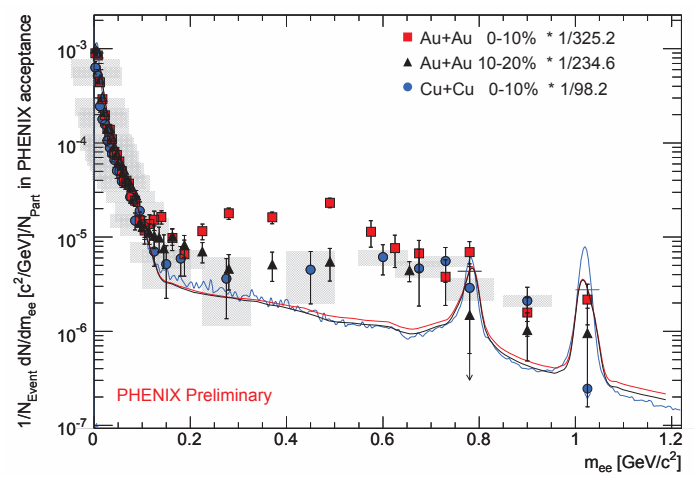

Fig. 5. The dielectron mass spectra for central heavy ion collisions scaled by the inverse of the $N_{\text {Part }}$. The $0-10 \% \mathrm{Au}+\mathrm{Au}$ are shown as red squares, the $10-20 \%$ $\mathrm{Au}+\mathrm{Au}$ are shown as black triangles and the $0-10 \%$ $\mathrm{Cu}+\mathrm{Cu}$ are shown as blue circles. The scaled cocktails for each of these spectra are presented in their respective colors.[16] [20]

$\mathrm{d}+\mathrm{Au}, \mathrm{Cu}+\mathrm{Cu}$ and $\mathrm{Au}+\mathrm{Au}$ spectra. The small excess at low mass and high $p_{T}$ in $\mathrm{p}+\mathrm{p}$ collisions is fully described by a direct photon component that is consistent with NLO pQCD calculations. In MB $\mathrm{Au}+\mathrm{Au}$ the thermal photon measurement is extrapolated to find the initial temperature of the fireball, projecting values of 300 to $600 \mathrm{MeV}$ for thermalization times of 0.6 to $0.15 \mathrm{fm} / \mathrm{c}$ respectively [21].

The low mass region of the $\mathrm{MB} \mathrm{Au}+\mathrm{Au}$ dielectron continuum contains more modification than a photonic source can describe. A second source of mass modification exists at low mass and low $p_{T}$. The effective temperature of this low mass, low $p_{T}$ excess can be extracted by fitting the $m_{T}-m_{0}$ spectra in the mass region $0.3-0.75 \mathrm{GeV} / \mathrm{c}^{2}$ after the data has been corrected into the full $2 \pi$ angular acceptance, as seen in [16]. The $m_{T}$ spectrum has a kink with a much softer component below $0.6 \mathrm{GeV} / c^{2}$ and a harder trend above $0.6 \mathrm{GeV} / c^{2}$ corresponding to the two sources of the excess in this mass region. Fitting the low mass $m_{T}$ spectrum with two exponentials or an exponential plus the thermal photon component results in low effective temperatures of $92.0 \pm 11.4 \pm 8.4 \mathrm{MeV}$ and $86.5 \pm 12.7_{-28.4}^{+11} \mathrm{MeV}$ below $0.6 \mathrm{GeV} / c^{2}$. This suggests that the excess below $0.6 \mathrm{GeV} / c^{2}$, which is the majority of the excess, is very soft with a low effective temperature[16].

$\mathrm{A}$ variety of theoretical comparisons have been made to the $\mathrm{MB} \mathrm{Au}+\mathrm{Au}$ mass, $p_{T}$ and $m_{T}-m_{0}$ spectra including Rapp [22] [23], Dusling and Zahed [24], and Cassing and Bratkovskaya [25] [26]. The Rapp theory describes light vector mesons using the vector dominance model. Dusling and Zahed 

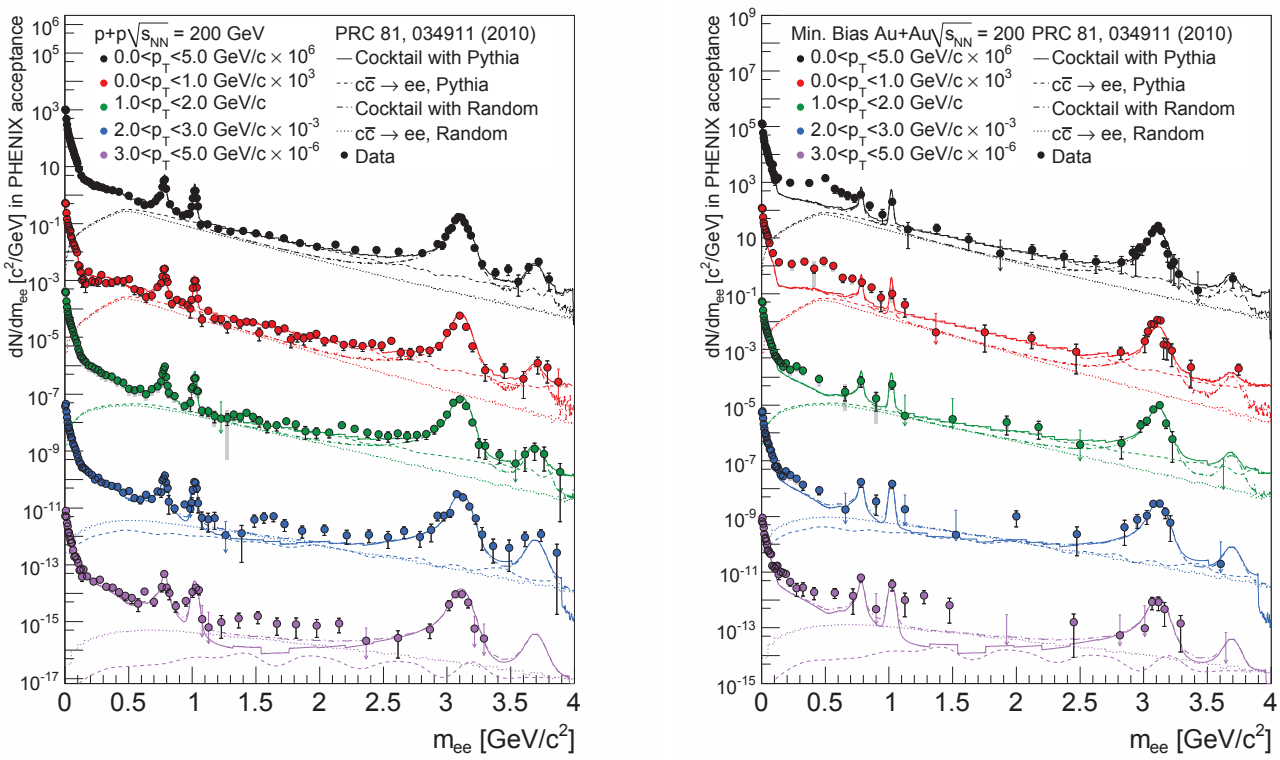

Fig. 6. The dielectron mass spectra of $\mathrm{p}+\mathrm{p}$ and $\mathrm{MB} \mathrm{Au}+\mathrm{Au}$ in the pair $p_{T}$ ranges: $0-5 \mathrm{GeV}$ (black), $0-1 \mathrm{GeV} / \mathrm{c}$ (red), $1-2 \mathrm{GeV} / \mathrm{c}$ (green), $2-3 \mathrm{GeV} / \mathrm{c}$ (blue) and 3-5 GeV/c (purple). The data are compared to their cocktail of the hadronic and charm contributions. A second cocktail using a randomly oriented $c-\bar{c}$ contribution is also shown as a dash-dot line.

use a chiral reduction formalism in vacuum and medium modifications are caused by of the hydrodynamic evolution. Cassing and Bratkovskaya applies a microscopic relativistic transport model. None of these theories are able to describe PHENIX's large low mass excess in MB Au+Au, particularly in the low $p_{T}$ region.

The STAR collaboration has measured the dielectron continuum in $\mathrm{p}+\mathrm{p}$ and $\mathrm{Au}+\mathrm{Au}$ collisions [27] [28]. The $p+p$ results are consistent with the STAR cocktail within systematic uncertainty. STAR's MB $\mathrm{Au}+\mathrm{Au}$ preliminary results have a smaller excess over the STAR cocktail in the low mass region, a factor of $1.53 \pm 0.07$ (stat) \pm 0.41 (sys) compared to the cocktail without the $\rho$ meson [28]. One issue when comparing STAR and PHENIX's excesses is the different acceptances in azimuth and rapidity of the two measurements. Another concern is the differences in the respective cocktails. STAR's cocktail excludes the $\rho$ meson, while PHENIX's cocktail includes it. The STAR cocktail uses a much larger the charm cross section, $0.92 \pm 0.10($ stat $) \pm 0.26($ sys $) \mathrm{mb}$ [27], which is almost twice the amount used in the PHENIX cocktail. These different cocktails are worrisome when interpreting results in the low mass region where the $\rho$ is located and the correlated charm component peaks.

\section{Conclusion}

The $\phi$ and $\omega$ mesons are suppressed in central and mid-central heavy ion collisions in the $p_{T}$ ranges 4-7 $\mathrm{GeV} / \mathrm{c}$ and $4-11 \mathrm{GeV} / \mathrm{c}$ respectively. They experience no strong cold nuclear effects in $\mathrm{d}+\mathrm{Au}$ collisions. In central $\mathrm{Au}+\mathrm{Au}$ collisions, the high $p_{T} \omega$ and $\phi$ are suppressed by a factor of five and coincide with the $\pi^{0}$ suppression patterns. This suggests that the meson suppression at high $p_{T}$ is caused by partonic energy loss. At intermediate $p_{T}, \phi$ 's are less suppressed than $\pi^{0}$ 's and agree with $K^{+}$suppression suggesting a recombination production mechanism. Extension of the $R_{A A}$ of the $K^{+}$to higher $p_{T}$ will shed more light on the $\phi$ production mechanism. Additionally, measuring the $\eta R_{A A}$ at intermediate $p_{T}$ will provide more information on the effect of strangeness enhancement since $\eta$ mesons have strange quark contributions. 
The PHENIX dielectron continuum shows a substantial low mass excess in central $\mathrm{Au}+\mathrm{Au}$ collisions. The low mass excess is also measured in the most central $\mathrm{Cu}+\mathrm{Cu}$ collisions and increases at a rate faster than $N_{\text {Part }}^{2}$ with centrality. While the high $p_{T}$, low mass excess is due to thermal virtual photons, the sizable low $p_{T}$, low mass excess has yet to be reproduced by theoretical calculations. The $\mathrm{p}+\mathrm{p}$ and $\mathrm{d}+\mathrm{Au}$ spectra agree with the cocktail of known dielectron sources suggesting no cold nuclear matter effects. Future measurements in PHENIX include the d+Au dielectron spectra in centrality bins and $\mathrm{p}+\mathrm{p}$ and $\mathrm{Au}+\mathrm{Au}$ analyses with the Hadron Blind Detector (HBD) detector. The HBD should improve the signal-to-background in the dielectron analysis by reducing the combinatorial background [29]. To understand the comparison of PHENIX and STAR's results, spectra with the same acceptance and cocktails with the same charm cross section are necessary. PHENIX's silicon vertex detector will help determine the correlated heavy quark contribution from data by identifying off-vertex electrons from open heavy flavor meson decays [30].

\section{References}

1. R. Rapp, Phys. Rev. C 63 (2001) 054907

2. R. Arnaldi et al., Phys. Rev. Lett. 96 (2006) 162302

3. A. Adare et al., Phys. Rev. Lett. 101 (2008) 232301

4. A. Adare et al., Phys. Rev. C 82 (2010) 011902

5. A. Adare et al., Phys. Rev. C 83 (2011) 064903

6. J. Adams et al., Phys. Rev. Lett. 92 (2004) 052302

7. J. Adams et al., Phys. Rev. C 71 (2005) 064902

8. K. Adcox et al., Nucl. Inst. and Meth. A 499 (2003) 489

9. M. Aizawa et al., Nucl. Inst. and Meth. A 499 (2003) 508

10. M. Allen et al., Nucl. Inst. and Meth. A 499 (2003) 549

11. A. Adare et. al., Phys. Rev. C 83 (2011) 024909

12. S. S. Adler et. al., Phys. Rev. C 75 (2007) 051902

13. C. Loizides, Eur. Phys. J. C 49 (2007) 339

14. M. Gyulassy, P. Levai and I. Vitev, Phys. Rev. Lett. 85 (2000) 5535

15. A. Adare et. al., Phys. Rev. C 84 (2011) 044902

16. A. Adare et. al., Phys. Rev. C 81 (2010) 02391

17. A. Adare et. al., Phys. Lett. B 670 (2009) 313

18. A. Adare, Phys. Rev. Lett. 97 (2006) 252002

19. Jason Kamin for the PHENIX Collaboration, J. Phys. G: Nucl. Part Phys. 38124181

20. Sarah Campbell for the PHENIX Collaboration, J. of Phys: Conf. Series 230012034

21. A Adare et. al., Phys. Rev. Lett. 104 (2010) 132301

22. W Lui and R. Rapp, Nucl. Phys. A 796 (2007) 101

23. Ralf Rapp, AIP Conf. Proc. 1322 (2010) 55

24. K. Dusling and I. Zahed, Nucl. Phys. A 825 (2009) 212

25. E. L. Bratkovskaya, W. Cassing and O. Linnyk, Phys. Lett. B 670 (2009) 428

26. O Linnyk, W. Cassing, J. Manninen, E. L. Bratkovskaya and C. M. Ko, arXiv 1111.2975

27. L. Adamczyk et al., arXiv:1204.1809

28. Jie Zhao for the STAR Collaboration, arXiv:1106.6146

29. A. Kozlov et al., Nucl. Inst. and Meth. A 636 (2011) 99

30. Johann Heuser et al., Nucl. Inst. and Meth. A 511 (2003) 210 